\title{
Article \\ Feeding Fiber-Bound Polyphenol Ingredients at Different Levels Modulates Colonic Postbiotics to Improve Gut Health in Dogs
}

\author{
Dennis E. Jewell ${ }^{1}{ }^{\mathbb{D}}$, Matthew I. Jackson ${ }^{2}$, Chun-Yen Cochrane ${ }^{2}$ and Dayakar V. Badri ${ }^{2, *}$ \\ 1 Department of Grain Science and Industry, Kansas State University, Manhattan, KS 66506, USA; \\ djewell@ksu.edu \\ 2 Hill's Pet Nutrition, Inc., Topeka, KS 66617, USA; matthew_jackson@hillspet.com (M.I.J.); \\ chun-yen_cochrane@hillspet.com (C.-Y.C.) \\ * Correspondence: dayakar_badri@hillspet.com
}

check for updates

Citation: Jewell, D.E.; Jackson, M.I.; Cochrane, C.-Y.; Badri, D.V. Feeding Fiber-Bound Polyphenol Ingredients at Different Levels Modulates Colonic Postbiotics to Improve Gut Health in Dogs. Animals 2022, 12, 627. https:// doi.org/10.3390/ani12050627

Academic Editor: Mandy Paterson

Received: 29 January 2022

Accepted: 1 March 2022

Published: 2 March 2022

Publisher's Note: MDPI stays neutral with regard to jurisdictional claims in published maps and institutional affiliations.

Copyright: (c) 2022 by the authors. Licensee MDPI, Basel, Switzerland. This article is an open access article distributed under the terms and conditions of the Creative Commons Attribution (CC BY) license (https:// creativecommons.org/licenses/by/ $4.0 /)$.

\begin{abstract}
Simple Summary: Microbes present in the large intestine of humans and companion animals produce bioactive metabolites from host-ingested food. These bioactive metabolites can influence host health. A prior study in dogs that were healthy or had chronic enteritis/gastroenteritis showed that stool quality improved when they ate food containing a fiber bundle made from fibers of pecan shells, flax seed, cranberry, citrus, and beet. In addition, eating food containing the fiber bundle resulted in the gut bacteria shifting from digesting mainly protein to digesting mainly carbohydrates. The present study tested the impact of the fiber bundle at a lower range of concentrations in dogs. Fecal levels of several bioactive metabolites with beneficial antioxidant or anti-inflammatory properties increased after dogs consumed food with the fiber bundle, though no changes in the bacteria or their functional pathways were observed. Stool quality remained in the acceptable range. These results suggest that the gut bacteria were able to digest the fiber bundle to produce beneficial bioactive metabolites to improve host health.
\end{abstract}

Abstract: This study assessed changes in canine fecal metabolites and microbiota with the consumption of foods with increasing concentrations of a fiber bundle including pecan shells, flax seed, and powders of cranberry, citrus, and beet that was previously shown (at $14 \% w / w$ ) to improve stool quality, shift fecal bacterial metabolism from proteolysis to saccharolysis, increase abundance of saccharolytic bacteria, and decrease abundance of proteolytic bacteria. In this study, 48 healthy adult dogs were split evenly to consume different inclusion levels $(0 \%, 1 \%, 2 \%$, and $4 \%)$ of the fiber bundle for a 31-day period following a 28-day pre-feed period. Increases from baseline in the fecal short-chain fatty acids butyric acid, valeric acid, and hexanoic acid were observed only in the dogs that consumed the food with the $4 \%$ fiber bundle. With addition of any level of the fiber bundle, increases were seen in the polyphenols hesperidin, hesperetin, ponciretin, secoisolariciresinol diglucoside, secoisolariciresinol, and enterodiol. However, fecal microbiota and their metabolism, and stool scores were largely unaffected by the fiber bundle. Overall, addition of the fiber bundle appeared to increase bioactive metabolites of increased antioxidant and anti-inflammatory potency for beneficial to health and, at levels $\geq 4 \%$, shifted gut bacterial metabolism toward saccharolysis.

Keywords: canine; fiber bundle; bioactive metabolites; microbiota; anti-inflammatory; short-chain fatty acids

\section{Introduction}

The interplay among nutrition, the gut microbiota and its metabolites, and host health has been the subject of numerous studies over the last decade [1,2]. Dietary consumption of fiber is known to yield health benefits, and many fiber-rich foods are also high in polyphenols. Most dietary polyphenols arrive largely intact to the large intestine, where they are 
metabolized by intestinal microbiota [3], and these postbiotic compounds are proposed to confer many of the observed beneficial health effects [4]. Further, these compounds appear to modulate the gut microbiota, with a decrease in pathogenic bacteria $[4,5]$.

Consumption of high-fiber foods also results in the increase of short-chain fatty acids (SCFAs), which are used as energy sources for colonocytes, contribute to maintenance of the intestinal tight junction barrier, and decrease inflammation [6,7]. An increase in SCFAs is also considered to be beneficial since they are decreased in people with ulcerative colitis or Crohn disease [8].

Several studies have examined the role of various added fibers in canine food in changing the gut microbiota and/or metabolites [9-16]. One of these prior studies tested a fiber bundle composed of pecan shells, flax seed, and cranberry, citrus, and beet powders to provide lignin as an insoluble bulking fiber, moderately fermentable fibers (whole flax), and fermentable fibers (e.g., hemicellulose and pectin) [16]. The change in fecal metabolites with the addition of the fiber bundle appeared to shift metabolism from a proteolytic state toward saccharolysis when added to either hydrolyzed meat or grain-rich foods in dogs that were healthy or had chronic enteritis/gastroenteritis [16]. In addition, the abundance of saccharolytic bacteria increased and proteolytic bacteria decreased with inclusion of the fiber bundle. Stool quality [17] also improved in both healthy dogs and those with chronic enteritis/gastroenteritis following consumption of food with the fiber bundle [16].The present study extended the original study by testing the fiber-bound polyphenolic ingredients of the fiber bundle at different levels in a single type of canine food (grain-rich), with the purpose of testing the differential impact on fecal metabolites and microbiota as availability of substrates for fermentation decreased.

\section{Materials and Methods}

\subsection{Study Foods}

All foods were manufactured at the Hill's experimental food laboratory. The control food used in this study was the same as the grain-rich control food used in a previous study [16]. The test foods were of similar formulation to the control food except they were supplemented with the fiber bundle (composed of ground pecan shells, dried pelleted beet pulp, ground citrus pulp, whole brown flax seed, and cranberry pomace) at $1 \%, 2 \%$, or $4 \% w / w$ on a dry matter basis and replaced all or some of the cellulose in the control food. Compositions of the foods, all in dry form, were formulated to canine adult maintenance standards and were equivalent across control and test foods (Table 1) to ensure that any differences observed were due to the addition of the fiber bundle rather than perturbation of macronutrients. Food formulations are shown in Table S1. All foods met the maintenance nutrition requirements of the Association of American Feed Control Officials.

\subsection{Animals and Experimental Design}

The study protocol was approved by the Hill's Institutional Animal Care and Use Committee (IACUC; CP848a.0.0.0-A-C-D-ADH-MULTI-98-GI). This study complied with the guide for the care and use of laboratory animals from the US National Research Council [18].

A total of 48 healthy adult dogs ( 24 female, 24 male), all spayed or neutered and owned by Hill's Pet Nutrition, Inc., were included. Dogs were housed at the Pet Nutrition Center in pairs with regular access to natural light, daily exercise, and socialization opportunities. All dogs were fed the control food for 28 days and then divided into four groups of 12 dogs each based on their sex, age, and body weight (Figure 1). Each group continued into the treatment phase in a parallel experimental design with consumption of the control food or foods with the fiber bundle added to $1 \%, 2 \%$, or $4 \%$ for 31 days. Feeding each dog the control food prior to the treatment food phase should reduce the individual effect in the later microbiota analysis. Each dog was fed based on their caloric requirements as calculated by their body weight. All dogs were healthy at the end of the study, with no adverse events reported. 
Table 1. Composition of test foods containing $0,1,2$, or $4 \%$ added fiber-bound polyphenol ingredients (grams/100 grams as mixed or as fed, unless otherwise stated).

\begin{tabular}{|c|c|c|c|c|}
\hline \multirow[b]{2}{*}{ Nutrient Parameter } & \multirow[b]{2}{*}{ Control Food } & \multicolumn{3}{|c|}{ Fiber Bundle Percentage in Food } \\
\hline & & $1 \%$ & $2 \%$ & $4 \%$ \\
\hline Moisture & 8.11 & 8.38 & 8.50 & 8.41 \\
\hline Protein, crude & 23.81 & 23.44 & 23.56 & 23.63 \\
\hline Fat & 13.16 & 14.27 & 12.95 & 13.01 \\
\hline Atwater energy ${ }^{1}(\mathrm{kcal} / \mathrm{kg})$ & 3569 & 3631 & 3567 & 3588 \\
\hline Ash & 5.81 & 5.96 & 5.98 & 5.85 \\
\hline Crude fiber & 2.4 & 2.3 & 2.1 & 1.8 \\
\hline Total dietary fiber & 6.1 & 6.7 & 6.2 & 6.8 \\
\hline Total insoluble fiber & 4.6 & 5.1 & 5.7 & 5.3 \\
\hline Total soluble fiber & 1.5 & 1.6 & 0.5 & 1.5 \\
\hline Calcium & 1.02 & 1.00 & 1.06 & 1.04 \\
\hline Phosphorus & 0.75 & 0.77 & 0.79 & 0.78 \\
\hline Sodium & 0.40 & 0.40 & 0.41 & 0.40 \\
\hline Capric acid (10:0) & $<0.02$ & $<0.02$ & $<0.02$ & $<0.02$ \\
\hline Lauric acid (12:0) & $<0.02$ & $<0.02$ & $<0.02$ & $<0.02$ \\
\hline Myristic acid (14:0) & 0.07 & 0.07 & 0.07 & 0.07 \\
\hline Palmitic acid (16:0) & 2.92 & 2.79 & 2.70 & 2.72 \\
\hline Palmitoleic acid (16:1) & 0.70 & 0.66 & 0.63 & 0.64 \\
\hline Steric acid (18:0) & 0.86 & 0.81 & 0.80 & 0.80 \\
\hline Oleic acid (18:1) & 4.68 & 4.45 & 4.33 & 4.38 \\
\hline Arachidic acid (20:0) & 0.02 & $<0.02$ & 0.02 & $<0.02$ \\
\hline LA $(18: 2(n-6))$ & 2.69 & 2.73 & 2.71 & 2.74 \\
\hline aLA $(18: 3(n-3))$ & 0.18 & 0.22 & 0.27 & 0.35 \\
\hline ARA $(20: 4(n-6))$ & 0.05 & 0.05 & 0.05 & 0.05 \\
\hline EPA $(20: 5(n-3))$ & $<0.02$ & $<0.02$ & $<0.02$ & $<0.02$ \\
\hline DHA $(22: 6(n-3))$ & $<0.02$ & $<0.02$ & $<0.02$ & $<0.02$ \\
\hline Lysine & 1.10 & 1.08 & 1.10 & 1.09 \\
\hline Threonine & 0.89 & 0.85 & 0.84 & 0.85 \\
\hline Methionine & 0.53 & 0.54 & 0.53 & 0.54 \\
\hline Cystine & 0.31 & 0.31 & 0.31 & 0.31 \\
\hline Tryptophan & 0.27 & 0.27 & 0.27 & 0.29 \\
\hline
\end{tabular}

${ }^{1}$ Calculated from analyticals using modified Atwater numbers (kcal/g of 3.5 for protein, 8.5. for fat, and 3.5 for nitrogen-free extract). aLA, alpha-linolenic acid; ARA, arachidonic acid; DHA, docosahexaenoic acid; EPA, eicosapentaenoic acid; LA, linoleic acid.

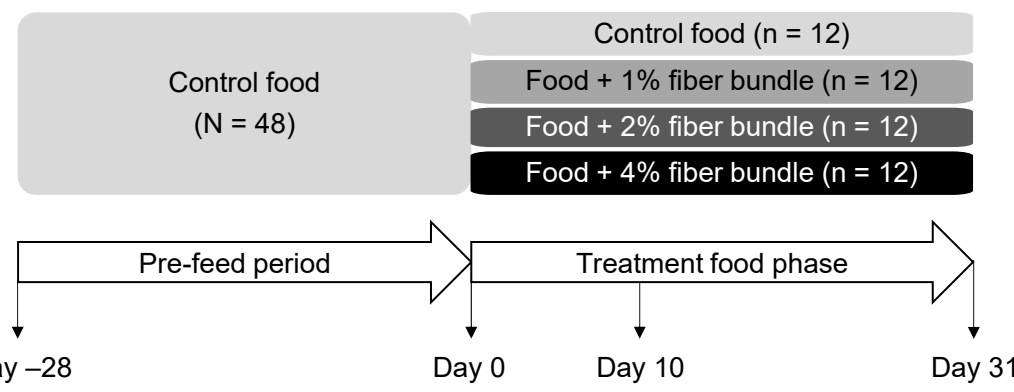

Figure 1. Study design and timeline in which dogs consumed foods containing $0 \%, 1 \%, 2 \%$, or $4 \%$ of the fiber bundle for 31 days. Blood and fecal samples were collected at days 0,10 , and 31 of the treatment food phase.

Blood/serum and feces from each dog were collected at the end of pre-feed phase and at days 10 and 31 days in the treatment phase. Dogs were sedated before phlebotomy. After collecting the blood samples in serum separator tubes (SST ${ }^{\mathrm{TM}}$ Serum Separation Tubes, Fisher Scientific, Waltham, MA, USA), samples remained at room temperature for $20 \mathrm{~min}$ to clot and were then centrifuged for $10 \mathrm{~min}$ at room temperature to separate serum from blood cells. Collected serum was aliquoted and stored at $-70{ }^{\circ} \mathrm{C}$ for various analyses. One fecal sample per dog was collected within $30 \mathrm{~min}$ of defecation.

\subsection{Stool Scoring and Fecal Sample Processing}

Fecal scores were determined on a 1-5 scale where grade 1: no solid form and $>75 \%$ liquid, grade 2 : soft and 50\% solid and 50\% liquid, grade 3: some cylindrical shape and $>75 \%$ formed and solid, grade $4:>75 \%$ cylindrical and $>50 \%$ firm, and grade 5 : cylindrically shaped and $>80 \%$ firm [17]. Each fecal sample was then homogenized using a Thinky Mixer (Thinky USA, Inc., Laguna Hills, CA, USA) following the Hill's Pet Nu- 
trition protocol as previously described [16]. Fecal $\mathrm{pH}$ was measured immediately after homogenization. Homogenized fecal samples were frozen at $-70{ }^{\circ} \mathrm{C}$ until analysis.

\subsection{Serum and Metabolite Analyses}

Blood count profiles (Sysmex XN 1000-V, Sysmex America, Inc., Lincolnshire, IL, USA) and serum chemistry (Cobas c501, Roche Diagnostics, Indianapolis, IN, USA) were analyzed according to manufacturers' instructions. Fecal SCFAs and metabolites were analyzed by Metabolon, Inc. (Morrisville, NC, USA).

\subsection{Fecal Microbiome Analysis and Bioinformatics Processing}

Fecal microbiome analysis was performed utilizing the Hill's Pet Nutrition protocols [19]. Briefly, total DNA was extracted from frozen feces samples using the PowerFecal DNA isolation kit (MO BIO, Carlsbad, CA, USA) according to the manufacturer's instructions with the modification of introducing a sonication step before vortexing the bead tubes with fecal samples horizontally for $15 \mathrm{~min}$. PCR amplification used the primer pairs spanning the V3-V4 hypervariable regions of the 16S rRNA gene. Sequencing was performed using the Illumina (San Diego, CA, USA) MiSeq platform, and the resulting sequences were de-multiplexed based on the dual index sequences by employing the MiSeq built-in metagenomics workflow to obtain FASTQ files. FASTQ sequence files were processed using the standard parameters of the Mothur software [20] and taxonomic classification was obtained by using the Greengenes reference database [21]. Operational taxonomic units (OTUs) were identified based on taxonomic hierarchy and further processed using the Phylogenetic Investigation of Communities by Reconstruction of Unobserved States (PICRUSt) protocol [22] to correct for copy numbers of the $16 \mathrm{~S}$ genes in their respective taxa, followed by predicting functional attributes using the Kyoto Encyclopedia of Genes and Genomes (KEGG) database.

\subsection{Statistical Analysis}

Fecal microbiome data were analyzed at the phylum, family, and genera levels. Only the OTUs and the PICRUSt-predicted KEGG ortholog functions present in at least $70 \%$ of all the fecal samples were considered in the following statistical analysis. The individual OTU counts (corrected for $16 \mathrm{~S}$ copy number) were analyzed by a negative binomial mixed model to study the effects of food treatments and collection time-points. The model also included a pre-feed covariate and a random animal factor. Permutational multivariate analysis of variance (PERMANOVA) based on the Manhattan distance of relative abundances was used to compare microbial compositions and custom-curated KEGG orthlog functional compositions among food treatments. All $p$ values were adjusted for false discovery rate (FDR) using the Benjamini-Hochberg procedure. A principal coordinate analysis plot was made to visualize the proximity of the four food treatments on Day 31 using the Manhattan distance of the fecal microbial composition at the genus level.

For the metabolite analysis, all values were transformed to natural logs and the change was calculated by subtracting initial values from final values. Mean differences and change over time were evaluated by the Proc Mixed procedure of SAS 9.4. Values represented for fecal metabolites are in relative fold differences. Unlike the absolute values presented for other endpoints (e.g., SCFA), which can be compared to previously published reports, relative fold differences in metabolomics have meaning only in terms of change or differences of responsiveness between groups. Thus, fecal metabolite analyses report the significance of change from baseline (Day 0) and the degree to which those changes were significantly different among treatment groups.

\section{Results}

\subsection{Animal Demographics, Intakes, and Clinical Assessment}

Of the 48 healthy adult dogs in this study, the mean \pm SD age was $4.9 \pm 2.3$ years (range, 1.2-8.6 years). The mean body weight at baseline was $10.6 \pm 1.8 \mathrm{~kg}$ (range, 
7.7-14.5 kg) and was similar among all groups (Table 2). All dogs were fed the control food for 28 days prior to being divided evenly into four groups that were fed the control food or test foods containing $1 \%, 2 \%$, or $4 \%$ of the fiber bundle for 31 days. Food intake was similar among groups (Table 2). After 31 days of the food treatment period, slightly greater weight loss from baseline was observed in the control and $1 \%$ fiber bundle groups but not in the $2 \%$ and $4 \%$ fiber bundle groups $(-0.3 \mathrm{~kg}$ and $-0.1 \mathrm{~kg}$; Table 2). Serum albumin, urea nitrogen, triglycerides, and cholesterol were similar among groups, with no significant differences from baseline or among groups. A significant decrease was seen in serum total protein in the $1 \%$ group compared with baseline, as were significant increases from baseline in creatinine in the $2 \%$ and $4 \%$ groups. However, levels of these circulating markers remained within clinically normal ranges.

Table 2. Body weight and selected serum biochemistry parameters at baseline, end of study, and change from baseline (Day 0 ) in dogs that consumed foods containing $0 \%, 1 \%, 2 \%$, or $4 \%$ of added fiber-bound polyphenol ingredients.

\begin{tabular}{|c|c|c|c|c|}
\hline \multirow[b]{2}{*}{ Parameter } & \multirow[b]{2}{*}{ Control Food } & \multicolumn{3}{|c|}{ Fiber Bundle Percentage in Food } \\
\hline & & $1 \%$ & $2 \%$ & $4 \%$ \\
\hline \multicolumn{5}{|l|}{ Body weight, $\mathrm{kg}$} \\
\hline Day 0 & $10.7 \pm 0.55$ & $10.6 \pm 0.55$ & $10.9 \pm 0.55$ & $10.5 \pm 0.55$ \\
\hline Day 31 & $10.4 \pm 0.56$ & $10.3 \pm 0.56$ & $10.8 \pm 0.56$ & $10.3 \pm 0.56$ \\
\hline Change & $-0.3 \pm 0.10^{1}$ & $-0.3 \pm 0.10^{1}$ & $-0.1 \pm 0.10$ & $-0.1 \pm 0.10$ \\
\hline Food intake, kcal/(body weight in kg) ${ }^{0.75}$ & $112 \pm 5.5$ & $107 \pm 5.5$ & $119 \pm 5.5$ & $109 \pm 5.5$ \\
\hline \multicolumn{5}{|l|}{ Albumin, mg/dL } \\
\hline Day 0 & $3.57 \pm 0.08$ & $3.62 \pm 0.08$ & $3.45 \pm 0.08$ & $3.51 \pm 0.08$ \\
\hline Day 31 & $3.68 \pm 0.08$ & $3.64 \pm 0.08$ & $3.52 \pm 0.08$ & $3.60 \pm 0.08$ \\
\hline Change & $0.12 \pm 0.04$ & $0.02 \pm 0.04$ & $0.08 \pm 0.04$ & $0.09 \pm 0.04$ \\
\hline \multicolumn{5}{|l|}{ Total protein, $\mathrm{mg} / \mathrm{dL}$} \\
\hline Day 0 & $5.58 \pm 0.09$ & $5.78 \pm 0.09$ & $5.54 \pm 0.09$ & $5.65 \pm 0.09$ \\
\hline Day 31 & $5.54 \pm 0.11$ & $5.60 \pm 0.11$ & $5.47 \pm 0.11$ & $5.64 \pm 0.11$ \\
\hline Change & $-0.04 \pm 0.06$ & $-0.17 \pm 0.06^{1}$ & $-0.08 \pm 0.06$ & $-0.01 \pm 0.06$ \\
\hline \multicolumn{5}{|l|}{ Urea nitrogen, $\mathrm{mg} / \mathrm{dL}$} \\
\hline Day 0 & $12.2 \pm 0.8$ & $13.1 \pm 0.8$ & $14.1 \pm 0.8$ & $12.5 \pm 0.8$ \\
\hline Day 31 & $11.5 \pm 0.8$ & $12.9 \pm 0.8$ & $13.9 \pm 0.8$ & $12.4 \pm 0.8$ \\
\hline Change & $-0.6 \pm 0.6$ & $-0.2 \pm 0.6$ & $-0.2 \pm 0.6$ & $-0.1 \pm 0.6$ \\
\hline \multicolumn{5}{|l|}{ Creatinine, $\mathrm{mg} / \mathrm{dL}$} \\
\hline Day 0 & $0.71 \pm 0.03$ & $0.70 \pm 0.03$ & $0.68 \pm 0.03$ & $0.72 \pm 0.03$ \\
\hline Day 31 & $0.75 \pm 0.04$ & $0.71 \pm 0.04$ & $0.73 \pm 0.04$ & $0.76 \pm 0.04$ \\
\hline Change & $0.03 \pm 0.02$ & $0.01 \pm 0.02$ & $0.06 \pm 0.02^{1}$ & $0.04 \pm 0.02^{1}$ \\
\hline \multicolumn{5}{|l|}{ Triglycerides, mg/dL } \\
\hline Day 0 & $63.2 \pm 5.6$ & $59.1 \pm 5.6$ & $64.2 \pm 5.6$ & $65.6 \pm 5.6$ \\
\hline Day 31 & $66.9 \pm 5.1$ & $62.7 \pm 5.1$ & $68.7 \pm 5.1$ & $61.6 \pm 5.1$ \\
\hline Change & $3.7 \pm 4.1$ & $3.7 \pm 4.1$ & $4.5 \pm 4.1$ & $-4.0 \pm 4.1$ \\
\hline \multicolumn{5}{|l|}{ Cholesterol, mg/dL } \\
\hline Day 0 & $195.1 \pm 14.2$ & $207.6 \pm 14.2$ & $191.2 \pm 14.2$ & $219.2 \pm 14.2$ \\
\hline Day 31 & $203.5 \pm 14.7$ & $211.9 \pm 14.7$ & $184.0 \pm 14.7$ & $222.4 \pm 14.7$ \\
\hline Change & $8.2 \pm 5.8$ & $4.3 \pm 5.8$ & $-7.2 \pm 5.8$ & $3.2 \pm 5.8$ \\
\hline
\end{tabular}

Values are least square means \pm standard errors. ${ }^{1}$ Significantly different $(p<0.05)$ from baseline (Day 0$)$.

\subsection{Fecal Parameters and Analytes}

Fecal moisture following consumption of the test foods significantly differed among groups at 31 days and differed from baseline within some individual foods, but these small changes were not considered to be physiologically relevant (Table 3). Fecal ammonium levels and $\mathrm{pH}$ were similar among all groups at baseline and showed no notable changes at the end of the 31-day feeding period. Stool scores were within acceptable ranges and showed no significant differences at any time points within the study.

Fecal SCFAs were assessed at baseline and days 10 and 31 of the feeding treatment phase. The straight-chain SCFAs butyric acid, valeric acid, and hexanoic acid were all significantly greater than baseline in feces from dogs in the $4 \%$ fiber bundle group (Table 4). Otherwise, the control groups and those with lower levels of the fiber bundle did not show significant changes in straight-chain or branched-chain SCFAs among groups or from their baseline values. 
Table 3. Fecal moisture, ammonium, and $\mathrm{pH}$ at baseline, end of study, and change from baseline (Day 0 ) and stool scores in dogs that consumed foods containing $0 \%, 1 \%, 2 \%$, or $4 \%$ of added fiber-bound polyphenol ingredients.

\begin{tabular}{|c|c|c|c|c|}
\hline \multirow[b]{2}{*}{ Parameter } & \multirow[b]{2}{*}{ Control Food } & \multicolumn{3}{|c|}{ Fiber Bundle Percentage in Food } \\
\hline & & $1 \%$ & $2 \%$ & $4 \%$ \\
\hline \multicolumn{5}{|l|}{ Moisture } \\
\hline Day 0 & $68.3 \pm 0.9$ & $69.8 \pm 0.9$ & $68.1 \pm 0.9$ & $67.8 \pm 0.9$ \\
\hline Day $10, \%$ of Day 0 & $103 \pm 1^{\mathrm{a} 1}$ & $98 \pm 1^{\mathrm{b}}$ & $101 \pm 1^{\mathrm{a}, \mathrm{b}}$ & $103 \pm 1^{\mathrm{a} 1}$ \\
\hline Day $31, \%$ of Day 0 & $105 \pm 1^{\mathrm{a} 1}$ & $100 \pm 1^{b}$ & $104 \pm 1^{\mathrm{a} 1}$ & $104 \pm 1^{\mathrm{a} 1}$ \\
\hline \multicolumn{5}{|l|}{ Ammonium, $\mathrm{mmol} / \mathrm{g}$} \\
\hline Day 0 & $0.040 \pm 0.002$ & $0.044 \pm 0.002$ & $0.046 \pm 0.002$ & $0.045 \pm 0.002$ \\
\hline Day $10, \%$ of Day 0 & $97 \pm 9$ & $107 \pm 9$ & $94 \pm 9$ & $95 \pm 9$ \\
\hline Day $31, \%$ of Day 0 & $107 \pm 9$ & $110 \pm 9$ & $102 \pm 9$ & $104 \pm 9$ \\
\hline \multicolumn{5}{|l|}{$\mathrm{pH}$} \\
\hline Day 0 & $5.88 \pm 0.06$ & $5.92 \pm 0.06$ & $5.90 \pm 0.06$ & $5.97 \pm 0.06$ \\
\hline Day $10, \%$ of Day 0 & $100 \pm 1$ & $100 \pm 1$ & $100 \pm 1$ & $99 \pm 1$ \\
\hline Day $31, \%$ of Day 0 & $99 \pm 1$ & $102 \pm 1$ & $100 \pm 1$ & $98 \pm 1$ \\
\hline \multicolumn{5}{|l|}{ Stool score } \\
\hline Day 0 & $4.2 \pm 0.20$ & $4.2 \pm 0.19$ & $4.5 \pm 0.19$ & $4.0 \pm 1.19$ \\
\hline Day 10 & $4.3 \pm 0.19$ & $4.4 \pm 0.19$ & $4.7 \pm 0.19$ & $4.5 \pm 0.19$ \\
\hline Day 31 & $4.2 \pm 0.19$ & $4.4 \pm 0.19$ & $4.3 \pm 0.19$ & $4.5 \pm 0.19$ \\
\hline
\end{tabular}

Values are least square means \pm standard errors. ${ }^{1}$ Significantly different $(p<0.05)$ from baseline (Day 0$)$. Different superscripted letters represent significant differences within a row $(p<0.05)$.

Table 4. Fecal short-chain fatty acids at baseline (Day 0) and Days 10 and 31 in dogs that consumed foods containing $0 \%, 1 \%, 2 \%$, or $4 \%$ of added fiber-bound polyphenol ingredients.

\begin{tabular}{|c|c|c|c|c|}
\hline \multirow[b]{2}{*}{ SCFA } & \multirow[b]{2}{*}{ Control Food } & \multicolumn{3}{|c|}{ Fiber Bundle Percentage in Food } \\
\hline & & $1 \%$ & $2 \%$ & $4 \%$ \\
\hline \multicolumn{5}{|l|}{ Acetic acid } \\
\hline Day $0, \mu \mathrm{g} / \mathrm{g}$ & $4100 \pm 180$ & $4243 \pm 173$ & $4744 \pm 173$ & $4538 \pm 173$ \\
\hline Day $10 \%$ of Day 0 & $114 \pm 7$ & $103 \pm 7$ & $103 \pm 7$ & $104 \pm 7$ \\
\hline Day $31, \%$ of Day 0 & $112 \pm 7$ & $104 \pm 7$ & $93 \pm 7$ & $102 \pm 7$ \\
\hline \multicolumn{5}{|l|}{ Propionic acid } \\
\hline Day $0, \mu \mathrm{g} / \mathrm{g}$ & $3229 \pm 191$ & $3000 \pm 183$ & $3231 \pm 183$ & $3239 \pm 183$ \\
\hline Day $10, \%$ of Day 0 & $91 \pm 9$ & $104 \pm 9$ & $102 \pm 9$ & $96 \pm 9$ \\
\hline Day $31, \%$ of Day 0 & $86 \pm 9$ & $92 \pm 9$ & $90 \pm 9$ & $97 \pm 9$ \\
\hline \multicolumn{5}{|l|}{ Butyric acid } \\
\hline Day $0, \mu \mathrm{g} / \mathrm{g}$ & $3547 \pm 449$ & $3960 \pm 430$ & $3355 \pm 430$ & $3962 \pm 430$ \\
\hline Day $10, \%$ of Day 0 & $92 \pm 25$ & $102 \pm 24$ & $123 \pm 24$ & $104 \pm 24$ \\
\hline Day $31, \%$ of Day 0 & $120 \pm 25$ & $110 \pm 24$ & $126 \pm 24$ & $158 \pm 24^{1}$ \\
\hline \multicolumn{5}{|l|}{ Valeric acid } \\
\hline Day $0, \mu g / g$ & $530 \pm 162$ & $468 \pm 155$ & $640 \pm 155$ & $559 \pm 155$ \\
\hline Day $10, \%$ of initial & $100 \pm 80$ & $166 \pm 76$ & $160 \pm 76$ & $121 \pm 76$ \\
\hline Day $31, \%$ of initial & $141 \pm 80$ & $156 \pm 76$ & $189 \pm 76$ & $299 \pm 76^{1}$ \\
\hline \multicolumn{5}{|l|}{ Hexanoic acid } \\
\hline Day $0, \mu \mathrm{g} / \mathrm{g}$ & $27 \pm 9.8$ & $8 \pm 9.5$ & $30 \pm 9.5$ & $26 \pm 9.5$ \\
\hline Day $10, \%$ of Day 0 & $151 \pm 251$ & $316 \pm 240$ & $99 \pm 240$ & $107 \pm 240$ \\
\hline Day $31, \%$ of Day 0 & $120 \pm 251$ & $140 \pm 240$ & $167 \pm 240$ & $756 \pm 240^{1}$ \\
\hline \multicolumn{5}{|l|}{ 2-methylpropionic acid } \\
\hline Day $0, \mu g / g$ & $188 \pm 21$ & $215 \pm 20$ & $257 \pm 20$ & $253 \pm 20$ \\
\hline Day $10, \%$ of Day 0 & $108 \pm 12$ & $117 \pm 12$ & $95 \pm 12$ & $91 \pm 12$ \\
\hline Day $31, \%$ of Day 0 & $120 \pm 12$ & $115 \pm 12$ & $97 \pm 12$ & $98 \pm 12$ \\
\hline \multicolumn{5}{|l|}{ 2-methylbutyric acid } \\
\hline Day $0, \mu \mathrm{g} / \mathrm{g}$ & $137 \pm 14$ & $151 \pm 14$ & $181 \pm 14$ & $175 \pm 14$ \\
\hline Day $10, \%$ of Day 0 & $97 \pm 11$ & $113 \pm 11$ & $94 \pm 11$ & $91 \pm 11$ \\
\hline Day 31, \% of Day 0 & $107 \pm 11$ & $115 \pm 11$ & $92 \pm 11$ & $102 \pm 11$ \\
\hline \multicolumn{5}{|l|}{ 3-methylbutyric acid } \\
\hline Day $0, \mu \mathrm{g} / \mathrm{g}$ & $223 \pm 23$ & $229 \pm 22$ & $274 \pm 22$ & $275 \pm 22$ \\
\hline Day $10, \%$ of Day 0 & $99 \pm 12$ & $119 \pm 12$ & $102 \pm 12$ & $85 \pm 12$ \\
\hline Day $31, \%$ of Day 0 & $103 \pm 12$ & $109 \pm 12$ & $97 \pm 12$ & $102 \pm 12$ \\
\hline
\end{tabular}

${ }^{1}$ Significantly different $(p<0.05)$ from baseline (Day 0$)$.

The concentrations of several dietary plant compounds and their postbiotics were significantly increased in feces from dogs fed any of the tested levels of the fiber bundle (Table 5). These included the polyphenols hesperidin, hesperetin, ponciretin, secoisolariciresinol diglucoside (SDG), secoisolariciresinol, and enterodiol. Arabinose was significantly increased in feces from the group that consumed the $4 \%$ fiber bundle food at both 
the day 10 and day 31 timepoints, while ribulose/xylulose was significantly increased in that group at day 10.

Table 5. Change from initial concentrations at Days 10 and 31 (natural log day 10 or $31-$ natural $\log$ Day 0 ) of selected fecal metabolites in dogs that consumed foods containing $0 \%, 1 \%, 2 \%$, or $4 \%$ of added fiber-bound polyphenol ingredients.

\begin{tabular}{|c|c|c|c|c|}
\hline \multirow[b]{2}{*}{ Metabolite } & \multirow[b]{2}{*}{ Control Food } & \multicolumn{3}{|c|}{ Fiber Bundle Percentage in Food } \\
\hline & & $1 \%$ & $2 \%$ & $4 \%$ \\
\hline \multicolumn{5}{|l|}{ Hesperidin } \\
\hline Day 10 ratio & $0.42 \pm 0.31^{\mathrm{a}}$ & $2.15 \pm 0.31^{\mathrm{b} 1}$ & $3.63 \pm 0.31^{\mathrm{c} 1}$ & $4.55 \pm 0.31^{\mathrm{d} 1}$ \\
\hline Day 31 ratio & $0.95 \pm 0.31^{\mathrm{a}}$ & $1.85 \pm 0.31^{\mathrm{b} 1}$ & $3.74 \pm 0.31^{\mathrm{c} 1}$ & $4.37 \pm 0.31^{\mathrm{c} 1}$ \\
\hline \multicolumn{5}{|l|}{ Hesperetin } \\
\hline Day 10 ratio & $0.62 \pm 0.38^{a}$ & $3.59 \pm 0.37^{\mathrm{b} 1}$ & $4.69 \pm 0.37^{\mathrm{c} 1}$ & $5.39 \pm 0.37^{\mathrm{c} 1}$ \\
\hline Day 31 ratio & $0.67 \pm 0.38^{a}$ & $3.22 \pm 0.37^{\mathrm{b} 1}$ & $4.33 \pm 0.37^{\mathrm{c} 1}$ & $5.24 \pm 0.37^{\mathrm{c} 1}$ \\
\hline \multicolumn{5}{|l|}{ Ponciretin } \\
\hline Day 10 ratio & $0.53 \pm 0.38^{a}$ & $3.19 \pm 0.36^{\mathrm{b} 1}$ & $3.93 \pm 0.36^{\mathrm{b} 1}$ & $4.76 \pm 0.36^{\mathrm{c} 1}$ \\
\hline Day 31 ratio & $0.51 \pm 0.38^{\mathrm{a}}$ & $2.82 \pm 0.36^{\mathrm{b} 1}$ & $3.56 \pm 0.36^{\mathrm{b} 1}$ & $4.75 \pm 0.36^{\mathrm{c} 1}$ \\
\hline \multicolumn{5}{|c|}{ Secoisolariciresinol diglucoside } \\
\hline Day 10 ratio & $0.01 \pm 0.24^{\mathrm{a}}$ & $1.41 \pm 0.23^{\mathrm{b} 1}$ & $1.85 \pm 0.23^{\mathrm{b}, \mathrm{c} 1}$ & $2.31 \pm 0.23^{\mathrm{c} 1}$ \\
\hline Day 31 ratio & $0.43 \pm 0.24^{\mathrm{a}}$ & $0.66 \pm 0.23^{\mathrm{a} 1}$ & $1.42 \pm 0.23^{\mathrm{b} 1}$ & $1.83 \pm 0.23^{b 1}$ \\
\hline \multicolumn{5}{|c|}{ Secoisolariciresinol } \\
\hline Day 10 ratio & $0.28 \pm 0.26^{\mathrm{a}}$ & $0.87 \pm 0.25^{\mathrm{b} 1}$ & $1.88 \pm 0.25^{\mathrm{c} 1}$ & $2.06 \pm 0.25^{\mathrm{c} 1}$ \\
\hline Day 31 ratio & $0.50 \pm 0.26^{\mathrm{a}}$ & $0.62 \pm 0.25^{\mathrm{a}, \mathrm{b} 1}$ & $1.17 \pm 0.25^{\mathrm{b}, \mathrm{c} 1}$ & $1.80 \pm 0.25^{\mathrm{cl}}$ \\
\hline \multicolumn{5}{|l|}{ Enterodiol } \\
\hline Day 10 ratio & $-0.47 \pm 0.30^{\mathrm{a}}$ & $0.91 \pm 0.29^{\mathrm{b} 1}$ & $0.99 \pm 0.29 \mathrm{~b} 1$ & $1.33 \pm 0.29^{b 1}$ \\
\hline Day 31 ratio & $0.32 \pm 0.30^{\mathrm{a}}$ & $0.97 \pm 0.29^{\mathrm{b} 1}$ & $1.00 \pm 0.29^{\mathrm{b} 1}$ & $2.07 \pm 0.29^{\mathrm{c} 1}$ \\
\hline \multicolumn{5}{|l|}{ Arabinose } \\
\hline Day 10 ratio & $0.50 \pm 0.15^{\mathrm{a} 1}$ & $0.14 \pm 0.14^{\mathrm{b}}$ & $0.22 \pm 0.14^{\mathrm{a}, \mathrm{b}}$ & $0.56 \pm 0.14^{\mathrm{a} 1}$ \\
\hline Day 31 ratio & $0.25 \pm 0.15^{\mathrm{a}}$ & $-0.26 \pm 0.14^{b}$ & $-0.23 \pm 0.14^{b}$ & $0.30 \pm 0.14^{\mathrm{a} 1}$ \\
\hline \multicolumn{5}{|c|}{ Ribulose/xylulose } \\
\hline Day 10 ratio & $0.12 \pm 0.11$ & $0.01 \pm 0.15$ & $0.22 \pm 0.14$ & $0.35 \pm 0.14^{1}$ \\
\hline Day 31 ratio & $0.17 \pm 0.11$ & $-0.01 \pm 0.15$ & $-0.10 \pm 0.14$ & $0.17 \pm 0.14$ \\
\hline
\end{tabular}

${ }^{1}$ Significantly different $(p<0.05)$ from baseline (Day 0). Different superscripted letters represent significant differences within a row $(p<0.05)$.

\subsection{Fecal Microbiota}

The principal coordinate analysis plot showed no clear separation in the fecal microbiome among any of the food types studied (Figure 2). Further, PERMANOVA analysis of the fecal microbiome composition at the phylum, family, and genera levels showed no significant differences among groups, nor did the curated KEGG ortholog function compositions (Table 6).



Figure 2. Principal coordinate analysis plot of the fecal microbiome of dogs that consumed foods containing $0 \%, 1 \%, 2 \%$, or $4 \%$ of added fiber-bound polyphenol ingredients for 31 days. 
Table 6. PERMANOVA analysis of bacterial taxa and KEGG pathways in feces from dogs that consumed the study foods.

\begin{tabular}{|c|c|c|c|c|}
\hline Microbiome & Food Comparison & Mean Squares & $p$-Value Corrected $*$ & $p$ Value \\
\hline \multicolumn{5}{|l|}{ Taxa } \\
\hline Phylum & All & 0.025 & 0.929 & 0.888 \\
\hline Family & All & 0.099 & 0.990 & 0.923 \\
\hline Genus & All & 0.131 & 0.981 & 0.969 \\
\hline \multicolumn{5}{|l|}{ KEGG pathways } \\
\hline Arginine & All & 0.0000009 & 0.989 & 0.916 \\
\hline Benzoate & All & 0.0000000 & 0.989 & 0.983 \\
\hline Butyrate & All & 0.0000025 & 0.989 & 0.976 \\
\hline Carbohydrate-active enzymes & All & 0.0000018 & 0.989 & 0.971 \\
\hline Phenylalanine & All & 0.0000002 & 0.989 & 0.912 \\
\hline Propionate & All & 0.0000016 & 0.989 & 0.928 \\
\hline Tryptophan & All & 0.0000000 & 0.989 & 0.946 \\
\hline Tyrosine & All & 0.0000002 & 0.989 & 0.903 \\
\hline
\end{tabular}

* By Benjamini-Hochberg method.

\section{Discussion}

A previous study demonstrated that inclusion of a fiber bundle at $14 \%$ in canine food significantly increased the abundance of saccharolytic gut bacteria, decreased the abundance of proteolytic bacteria, and also shifted gut microbial metabolism toward saccharolytic activity [16]. In the current study, we tested the same fiber bundle at various inclusion levels ranging from 1 to $4 \%$ while retaining the same ratios of fibrous ingredients in order to determine the lowest inclusion level that would manifest molecular signatures to potentially improve pet health. The inclusion of a fiber bundle in food appeared to lead to a shift toward saccharolytic metabolism of the gut microbiome of dogs fed the $4 \%$ fiber bundle food compared to $1 \%$ and $2 \%$, as indicated by the increased fecal levels of the SCFA butyrate and the sugar arabinose, which also suggests that gut microbiota could utilize the hemicellulose component of the fiber source. A number of other compounds with antioxidant and anti-inflammatory properties were present at greater levels in feces from dogs that consumed foods with any of the tested levels of the fiber bundle, consistent with the prior study in which the fiber bundle was included at 14\% [16]. Thus, although no significant changes in the composition of the gut microbiota were observed in this study, the results indicate that the fiber bundle led to a shift in their metabolism toward the production of beneficial compounds and potentially greater gut health.

It has been previously proposed that the interaction of polyphenols from food and the gut microbiota may positively influence host health [3]. Consumption, in humans, of flavonoids, a subset of polyphenols, has been shown to lower the risk of cancer, cardiovascular diseases, metabolic diseases, and neurodegenerative diseases [23,24]. In human trials, consumption of orange juice, which is rich in flavonoids, appeared to improve cognitive function in adults, and consumption of citrus fruits was inversely correlated with the risk of dementia in elderly participants [23].

Hesperidin, a flavonoid found in citrus fruits, was significantly increased with addition of the fiber bundle at all of the tested concentrations in this study. Supplementation with hesperidin has shown glucose-lowering effects and improvements in insulin resistance and inflammatory parameters in rodent models of both type 1 and type 2 diabetes [25,26]. Other cardiovascular benefits of hesperidin include its observed effects on lipid profiles, hypertension, oxidative stress, and inflammation $[25,26]$. The health benefits of hesperidin are attributed to its anti-inflammatory effects on cytokines and its antioxidant effects [26].

Hesperetin, which also significantly increased in feces following consumption of food with the fiber bundle, is metabolized from hesperidin by intestinal bacteria [27]. Hesperetin appears to have even greater bioavailability and anti-inflammatory and antioxidant properties than its precursor [26]. These benefits extend to diabetes, metabolic disorder, and other cardiovascular risk factors [25].

Hesperidin and hesperetin have both been observed to confer protective properties on neurons in both in vitro and animal models of various neurological diseases such as Alzheimer disease, epilepsy, Huntington disease, and Parkinson disease, in which cyto- 
toxicity was induced by inflammatory stimuli, oxidative stress, or neurotoxic stress in animal models $[23,28]$. In addition, hesperetin supplementation led to decreased oxidative stress, inflammation, and apoptosis in a murine model of myocardial ischemia [29]. Several flavonoids, including hesperetin, appear to increase the barrier integrity of the tight junction in human intestinal Caco-2 cells [30]. Extending this to an organismal level, several experiments in rodent models of chemically induced colitis have shown that hesperidin and hesperetin led to improvements in colitis symptoms, including inflammation and colonic barrier function [24].

Hesperetin, hesperidin, and other polyphenols in orange juice appear to stimulate the growth of beneficial bacteria and inhibit pathogenic bacteria in vitro [31,32]. Although a shift in the microbiome was not observed in the present study, this could have been due to the relatively low levels of inclusion of the fiber bundle. In the prior study that examined the effects of the fiber bundle at $14 \%$, the abundances of several genera of saccharolytic bacteria increased while those of potentially pathogenic bacteria decreased following consumption of the fiber bundle in either grain-rich or high-meat-food backgrounds [16].

Another flavonoid, ponciretin, is metabolized from poncirin, derived from citrus fruit, by intestinal bacteria, as shown in humans [27] and mice [33]. Like hesperidin, ponciretin has shown anticancer and anti-inflammatory effects, with cytotoxic effects on a colon cancer cell line in vitro [27] and attenuating colitis in mice by the anti-inflammatory effects of suppressing NF- $\mathrm{kB}$ activation and correcting the imbalance of Th17/Treg cells [33,34]. In addition, ponciretin can inhibit the growth of Helicobacter pylori [35], a bacterium implicated in gastritis, stomach ulcers, and lymphomas of the gastrointestinal tract [36].

The biological activity of SDG, the main lignan in flaxseed [37], results from its conversion to secoisolariciresinol, then enterodiol, and enterolactone, carried out by intestinal microbiota $[38,39]$. As in a prior study that tested the addition of low or high soluble fiber with betaine to food in canines, higher fecal levels of SDG and secoisolariciresinol were observed with increasing fiber consumption [40], as was enterodiol. Similar to polyphenols, the antioxidant and anti-inflammatory properties of SDG appear to translate to a number of positive health benefits in a multitude of diseases, including diabetes, cancer, and cardiovascular disease $[37,38]$. SDG exhibited positive effects in a variety of model systems, including antioxidant effects in cadmium-induced renal toxicity in rats [41], anti-inflammatory effects in the dextran sulfate sodium salt-induced colitis mouse model [42], and cytoprotective and anti-inflammatory effects in human umbilical vein endothelial cells [43]. SDG and enterodiol have shown apoptotic effects on cultured human colon carcinoma cells [44] and colorectal cancer cells in vitro [45], respectively.

The increases of the pentoses arabinose and ribulose/xylulose with the $4 \%$ fiber bundle food may have originated from breakdown of the dietary fiber. These sugars can then be fermented by the gut microbiota to produce SCFAs [46], which may also explain the increases in SCFAs observed with the $4 \%$ fiber bundle food in this study.

Several of the results seen here are consistent with those of other studies that have studied the effects of consumption of fiber and/or lignans. Increased butyric acid and arabinose have been observed with consumption of fiber in dogs compared with control foods $[15,16,47]$ and indicate a shift from proteolytic to saccharolytic metabolism. Dietary intake of lignans was also associated with higher levels of butyric acid in a human trial [48].

Interestingly, neither the fecal microbiome nor its functional pathways appeared to change to a significant extent with the addition of the fiber bundle in the present study, despite the changes to the metabolome. A recent review noted that food-induced changes in the microbiome of healthy dogs are not as extensive as the microbiome changes observed in disease states [49]. Since the compositions of the gut microbiota clearly shifted in the prior study in which the fiber bundle was included to $14 \%$ [16], there may be some minimum level required above the $4 \%$ used as the highest level in this study to observe changes in the microbiota. However, it is of interest that the metabolic signatures of the gut microbiome activity were altered at levels of fiber that were too low to induce measurable changes in the genetic signature of that same microbiome. 
A limitation of this study was that levels of the fiber bundle were perhaps too low to observe the greater shifts in metabolites and microbiota observed in the previous study with the fiber bundle included at $14 \%$ [16]. In addition, it is possible that greater effects would have been seen in the present study if the time had been extended beyond the 31-day feeding period, if more dogs per treatment group were included, or if dogs with an underlying condition, such as enteritis or gastroenteritis, were included. Further studies are needed to determine the optimal levels of different types of fiber for the production of beneficial metabolites to improve pet health.

\section{Conclusions}

In this study, a fiber bundle included in canine food at levels ranging from 1 to $4 \%$ was examined for its effects on fecal metabolites and microbiota in healthy dogs. There was virtually no effect on the microbiota at the end of the 31-day feeding period, in contrast to results from a prior study in which the fiber bundle was included at $14 \%$. However, levels of several metabolites with antioxidant and anti-inflammatory properties, including polyphenols and lignans, were increased with addition of the fiber bundle at $4 \%$, as were butyric acid and arabinose. Together, these indicate that the gut microbiota were able to utilize the fiber bundle toward saccharolytic metabolism and transform fiber-bound polyphenols into bioactive metabolites that increased the antioxidant and anti-inflammatory potency for a healthier state.

Supplementary Materials: The following supporting information can be downloaded at: https: / / www.mdpi.com/article/10.3390/ani12050627/s1, Table S1: Formulations of the foods used in this study.

Author Contributions: Conceptualization, D.E.J., M.I.J. and D.V.B.; methodology, D.E.J., M.I.J. and D.V.B.; formal analysis, D.E.J., M.I.J., C.-Y.C. and D.V.B.; investigation, D.E.J., M.I.J. and D.V.B.; resources, D.E.J., M.I.J. and D.V.B.; data curation, D.E.J., M.I.J., C.-Y.C. and D.V.B.; writing-review and editing, D.E.J., M.I.J., C.-Y.C. and D.V.B.; project administration, D.E.J., M.I.J. and D.V.B.; funding acquisition, D.E.J., M.I.J. and D.V.B. All authors have read and agreed to the published version of the manuscript.

Funding: This research was funded by Hill's Pet Nutrition, Inc.

Institutional Review Board Statement: The study protocol was approved by the Hill's Pet Nutrition Institutional Animal Care and Use Committee CP848a.0.0.0-A-C-D-ADH-MULTI-98-GI).

Informed Consent Statement: Not applicable.

Data Availability Statement: Data are available in the paper and its supplemental files or by request from the corresponding author.

Acknowledgments: Jennifer L. Giel assisted with the writing and development of the manuscript.

Conflicts of Interest: Although the funder provided support in the form of salaries for D.V.B., M.I.J. and C-Y.C., the funder had no role in the design of the study; in the collection, analyses, or interpretation of data; in the writing of the manuscript, or in the decision to publish the results. The research was performed at the Pet Nutrition Center, Topeka, KS, USA, where these authors currently (D.V.B., M.I.J.) or formerly (D.E.J.) work.

\section{References}

1. Shock, T.; Badang, L.; Ferguson, B.; Martinez-Guryn, K. The interplay between diet, gut microbes, and host epigenetics in health and disease. J. Nutr. Biochem. 2021, 95, 108631. [CrossRef] [PubMed]

2. Wernimont, S.M.; Radosevich, J.; Jackson, M.; Ephraim, E.; Badri, D.V.; MacLeay, J.M.; Jewell, D.E.; Suchodolski, J.S. The effects of nutrition on the gastrointestinal microbiome of cats and dogs: Impact on health and disease. Front. Microbiol. 2020, 11, 1266. [CrossRef] [PubMed]

3. Abbas, M.; Saeed, F.; Anjum, F.M.; Afzaal, M.; Tufail, T.; Bashir, M.S.; Ishtiaq, A.; Hussain, S.; Suleria, H.A.R. Natural polyphenols: An overview. Int. J. Food Prop. 2017, 20, 1689-1699. [CrossRef]

4. Cardona, F.; Andrés-Lacueva, C.; Tulipani, S.; Tinahones, F.J.; Queipo-Ortuño, M.I. Benefits of polyphenols on gut microbiota and implications in human health. J. Nutr. Biochem. 2013, 24, 1415-1422. [CrossRef] 
5. Selma, M.V.; Espín, J.C.; Tomás-Barberán, F.A. Interaction between phenolics and gut microbiota: Role in human health. J. Agric. Food Chem. 2009, 57, 6485-6501. [CrossRef]

6. Van Der Beek, C.M.; DeJong, C.H.C.; Troost, F.J.; Masclee, A.A.M.; Lenaerts, K. Role of short-chain fatty acids in colonic inflammation, carcinogenesis, and mucosal protection and healing. Nutr. Rev. 2017, 75, 286-305. [CrossRef]

7. Ríos-Covián, D.; Ruas-Madiedo, P.; Margolles, A.; Gueimonde, M.; De Los Reyes-Gavilán, C.G.; Salazar, N. Intestinal short chain fatty acids and their link with diet and human health. Front. Microbiol. 2016, 7, 185. [CrossRef]

8. Martinez, K.B.; Leone, V.; Chang, E.B. Microbial metabolites in health and disease: Navigating the unknown in search of function. J. Biol. Chem. 2017, 292, 8553-8559. [CrossRef]

9. Middelbos, I.S.; Boler, B.M.V.; Qu, A.; White, B.A.; Swanson, K.S.; Fahey, G.C., Jr. Phylogenetic characterization of fecal microbial communities of dogs fed diets with or without supplemental dietary fiber using 454 pyrosequencing. PLoS ONE 2010, 5, e9768. [CrossRef]

10. Panasevich, M.R.; Kerr, K.R.; Dilger, R.N.; Fahey, G.C.; Guérin-Deremaux, L.; Lynch, G.L.; Wils, D.; Suchodolski, J.S.; Steer, J.M.; Dowd, S.E.; et al. Modulation of the faecal microbiome of healthy adult dogs by inclusion of potato fibre in the diet. Br. J. Nutr. 2014, 113, 125-133. [CrossRef]

11. Panasevich, M.R.; Serao, M.C.R.; De Godoy, M.R.C.; Swanson, K.; Guérin-Deremaux, L.; Lynch, G.L.; Wils, D.; Fahey, G.C., Jr.; Dilger, R.N. Potato fiber as a dietary fiber source in dog foods. J. Anim. Sci. 2013, 91, 5344-5352. [CrossRef]

12. Vanhoutte, T.; Huys, G.; De Brandt, E.; Fahey, J.G.C.; Swings, J. Molecular monitoring and characterization of the faecal microbiota of healthy dogs during fructan supplementation. FEMS Microbiol. Lett. 2005, 249, 65-71. [CrossRef] [PubMed]

13. Maria, A.P.J.; Ayane, L.; Putarov, T.C.; Loureiro, B.A.; Neto, B.P.; Casagrande, M.F.; Gomes, M.O.S.; Glória, M.B.A.; Carciofi, A.C The effect of age and carbohydrate and protein sources on digestibility, fecal microbiota, fermentation products, fecal IgA, and immunological blood parameters in dogs. J. Anim. Sci. 2017, 95, 2452-2466. [CrossRef] [PubMed]

14. Kröger, S.; Vahjen, W.; Zentek, J. Influence of lignocellulose and low or high levels of sugar beet pulp on nutrient digestibility and the fecal microbiota in dogs. J. Anim. Sci. 2017, 95, 1598-1605. [CrossRef] [PubMed]

15. Simpson, J.; Martineau, B.; Jones, W.; Ballam, J.; Mackie, R. Characterization of fecal bacterial populations in canines: Effects of age, breed and dietary fiber. Microb. Ecol. 2002, 44, 186-197. [CrossRef] [PubMed]

16. Jackson, M.I.; Jewell, D.E. Balance of saccharolysis and proteolysis underpins improvements in stool quality induced by adding a fiber bundle containing bound polyphenols to either hydrolyzed meat or grain-rich foods. Gut Microbes 2018, 10, 298-320. [CrossRef]

17. Hall, J.A.; Melendez, L.D.; Jewell, D.E. Using gross energy improves metabolizable energy predictive equations for pet foods whereas undigested protein and fiber content predict stool quality. PLoS ONE 2013, 8, e54405. [CrossRef]

18. National Research Council. Guide for the Care and Use of Laboratory Animals, 8th ed.; The National Academies Press: Washington, DC, USA, 2011; ISBN 978-0-309-38629-6. [CrossRef]

19. Ephraim, E.; Cochrane, C.-Y.; Jewell, D.E. Varying protein levels influence metabolomics and the gut microbiome in healthy adult dogs. Toxins 2020, 12, 517. [CrossRef]

20. Schloss, P.D.; Westcott, S.L.; Ryabin, T.; Hall, J.R.; Hartmann, M.; Hollister, E.B.; Lesniewski, R.A.; Oakley, B.B.; Parks, D.H.; Robinson, C.J.; et al. Introducing mothur: Open-source, platform-independent, community-supported software for describing and comparing microbial communities. Appl. Environ. Microbiol. 2009, 75, 7537-7541. [CrossRef]

21. DeSantis, T.Z.; Hugenholtz, P.; Larsen, N.; Rojas, M.; Brodie, E.L.; Keller, K.; Huber, T.; Dalevi, D.; Hu, P.; Andersen, G.L. Greengenes, a chimera-checked $16 \mathrm{~S}$ rRNA gene database and workbench compatible with ARB. Appl. Environ. Microbiol. 2006, 72, 5069-5072. [CrossRef]

22. Langille, M.G.I.; Zaneveld, J.; Caporaso, J.G.; McDonald, D.; Knights, D.; Reyes, J.A.; Clemente, J.C.; Burkepile, D.E.; Vega Thurber, R.L.; Knight, R.; et al. Predictive functional profiling of microbial communities using 16S rRNA marker gene sequences. Nat. Biotechnol. 2013, 31, 814-821. [CrossRef] [PubMed]

23. Scoditti, E. Neuroinflammation and neurodegeneration: The promising protective role of the citrus flavanone hesperetin. Nutrients 2020, 12, 2336. [CrossRef] [PubMed]

24. Stevens, Y.; Van Rymenant, E.; Grootaert, C.; Van Camp, J.; Possemiers, S.; Masclee, A.; Jonkers, D. The intestinal fate of citrus flavanones and their effects on gastrointestinal health. Nutrients 2019, 11, 1464. [CrossRef]

25. Mas-Capdevila, A.; Teichenne, J.; Domenech-Coca, C.; Caimari, A.; Del Bas, J.M.; Escoté, X.; Crescenti, A. Effect of hesperidin on cardiovascular disease risk factors: The role of intestinal microbiota on hesperidin bioavailability. Nutrients 2020, 12, 1488. [CrossRef] [PubMed]

26. Gandhi, G.R.; Vasconcelos, A.B.S.; Wu, D.-T.; Li, H.-B.; Antony, P.J.; Li, H.; Geng, F.; Gurgel, R.Q.; Narain, N.; Gan, R.-Y. Citrus flavonoids as promising phytochemicals targeting diabetes and related complications: A systematic review of in vitro and in vivo studies. Nutrients 2020, 12, 2907. [CrossRef]

27. Kim, D.-H.; Jung, E.-A.; Sohng, I.-S.; Han, J.-A.; Kim, T.-H.; Han, M.J. Intestinal bacterial metabolism of flavonoids and its relation to some biological activities. Arch. Pharmacal. Res. 1998, 21, 17-23. [CrossRef]

28. Khan, A.; Ikram, M.; Hahm, J.R.; Kim, M.O. Antioxidant and anti-inflammatory effects of citrus flavonoid hesperetin: Special focus on neurological disorders. Antioxidants 2020, 9, 609. [CrossRef] 
29. Liu, P.; Li, J.; Liu, M.; Zhang, M.; Xue, Y.; Zhang, Y.; Han, X.; Jing, X.; Chu, L. Hesperetin modulates the Sirt1/Nrf2 signaling pathway in counteracting myocardial ischemia through suppression of oxidative stress, inflammation, and apoptosis. Biomed. Pharmacother. 2021, 139, 111552. [CrossRef]

30. Noda, S.; Tanabe, S.; Suzuki, T. Differential effects of flavonoids on barrier integrity in human intestinal Caco-2 cells. J. Agric. Food Chem. 2012, 60, 4628-4633. [CrossRef]

31. Duda-Chodak, A. The inhibitory effect of polyphenols on human gut microbiota. J. Physiol. Pharmacol. Off. J. Pol. Physiol. Soc. 2012, 63, 497-503.

32. Duque, A.L.R.F.; Monteiro, M.; Adorno, M.A.T.; Sakamoto, I.K.; Sivieri, K. An exploratory study on the influence of orange juice on gut microbiota using a dynamic colonic model. Food Res. Int. 2016, 84, 160-169. [CrossRef]

33. Kang, G.-D.; Kim, D.-H. Poncirin and its metabolite ponciretin attenuate colitis in mice by inhibiting LPS binding on TLR4 of macrophages and correcting Th17/Treg imbalance. J. Ethnopharmacol. 2016, 189, 175-185. [CrossRef] [PubMed]

34. Kang, G.-D.; Kim, D.-H. Ponciretin attenuates ethanol-induced gastric damage in mice by inhibiting inflammatory responses. Int Immunopharmacol. 2017, 43, 179-186. [CrossRef]

35. Kim, D.-H.; Bae, E.-A.; Han, M.J. Anti-Helicobacter pylori activity of the metabolites of poncirin from Poncirus trifoliata by human intestinal bacteria. Biol. Pharm. Bull. 1999, 22, 422-424. [CrossRef] [PubMed]

36. Padda, J.; Khalid, K.; Cooper, A.C.; Jean-Charles, G. Association between Helicobacter pylori and gastric carcinoma. Cureus 2021, 13, e15165. [CrossRef] [PubMed]

37. Kezimana, P.; Dmitriev, A.A.; Kudryavtseva, A.V.; Romanova, E.V.; Melnikova, N.V. Secoisolariciresinol diglucoside of flaxseed and its metabolites: Biosynthesis and potential for nutraceuticals. Front. Genet. 2018, 9, 641. [CrossRef]

38. Imran, M.; Ahmad, N.; Anjum, F.M.; Khan, M.K.; Mushtaq, Z.; Nadeem, M.; Hussain, S. Potential protective properties of flax lignan secoisolariciresinol diglucoside. Nutr. J. 2015, 14, 1-7. [CrossRef]

39. Senizza, A.; Rocchetti, G.; Mosele, J.I.; Patrone, V.; Callegari, M.L.; Morelli, L.; Lucini, L. Lignans and gut microbiota: An interplay revealing potential health implications. Molecules 2020, 25, 5709. [CrossRef]

40. Ephraim, E.; Jewell, D.E. Effect of added dietary betaine and soluble fiber on metabolites and fecal microbiome in dogs with early renal disease. Metabolites 2020, 10, 370. [CrossRef]

41. Aqeel, T.; Gurumallu, S.C.; Bhaskar, A.; Hashimi, S.M.; Javaraiah, R. Secoisolariciresinol diglucoside protects against cadmiuminduced oxidative stress-mediated renal toxicity in rats. J. Trace Elements Med. Biol. 2020, 61, 126552. [CrossRef]

42. Wang, Z.; Chen, T.; Yang, C.; Bao, T.; Yang, X.; He, F.; Zhang, Y.; Zhu, L.; Chen, H.; Rong, S.; et al. Secoisolariciresinol diglucoside suppresses Dextran sulfate sodium salt-induced colitis through inhibiting NLRP1 inflammasome. Int. Immunopharmacol. 2019, 78, 105931. [CrossRef] [PubMed]

43. Zhang, S.; Cheng, M.; Wang, Z.; Liu, Y.; Ren, Y.; Rong, S.; Wang, X. Secoisolariciresinol diglucoside exerts anti-inflammatory and antiapoptotic effects through inhibiting the akt/ikb/nf-kb pathway on human umbilical vein endothelial cells. Mediat. Inflamm. 2020, 2020, 1-8. [CrossRef] [PubMed]

44. Özgöçmen, M.; Bayram, D.; Türel, G.Y.; Toğay, V.A.; Calapoğlu, N. Secoisolariciresinol diglucoside induces caspase-3-mediated apoptosis in monolayer and spheroid cultures of human colon carcinoma cells. J. Food Biochem. 2021, 45, e13719. [CrossRef]

45. Shin, M.-K.; Jeon, Y.-D.; Jin, J.-S. Apoptotic effect of enterodiol, the final metabolite of edible lignans, in colorectal cancer cells. J. Sci. Food Agric. 2018, 99, 2411-2419. [CrossRef]

46. Basen, M.; Kurrer, S.E. A close look at pentose metabolism of gut bacteria. FEBS J. 2020, 288, 1804-1808. [CrossRef] [PubMed]

47. Thornton, E.; Robinson, E.; Templeman, J.R.; Bruggink, L.; Bower, M.; Cant, J.P.; Holloway, G.P.; Swanson, K.S.; Squires, E.J.; Shoveller, A.K. Supplemental fiber affects body temperature and fecal metabolites but not respiratory rate or body composition in mid-distance training sled dogs. Front. Vet. Sci. 2021, 8, 639335. [CrossRef]

48. Fernandez-Navarro, T.; Salazar, N.; Gutiérrez-Díaz, I.; Sánchez, B.; Rúas-Madiedo, P.; Reyes-Gavilan, C.D.L.; Margolles, A.; Gueimonde, M.; González, S. Bioactive compounds from regular diet and faecal microbial metabolites. Eur. J. Nutr. 2018, 57, 487-497. [CrossRef] [PubMed]

49. Pilla, R.; Suchodolski, J.S. The gut microbiome of dogs and cats, and the influence of diet. Vet. Clin. N. Am. Small Anim. Pract. 2021, 51, 605-621. [CrossRef] 